\title{
Obstruktion der großen Atemwege bei Colitis ulcerosa - eine seltene extraintestinale Manifestation
}

\author{
Central Airway Obstruction in Ulcerative Colitis - \\ A Rare Extraintestinal Manifestation
}

Autoren

Institute

\author{
A. Scherff', B. Khanavkar' ${ }^{1}$, U. Ostendorf', S. Phillipou ${ }^{2}$, S. Ewig
}

Thoraxzentrum Ruhrgebiet, Kliniken für Pneumologie, Beatmungsmedizin und Infektiologie,
Evangelisches Krankenhaus Herne und Augusta-Kranken-Anstalt Bochum
Institut für Pathologie, Augusta-Kranken-Anstalt Bochum eingereicht 2. 6. 2006

akzeptiert nach Revision

14. 6.2006

Bibliografie

Dol $10.1055 / \mathrm{s}-2006-944249$

Pneumologie 2006; 60; 607-610

(c) Georg Thieme Verlag KG

Stuttgart · New York

ISSN 0934-8387

\section{Korrespondenzadresse}

Prof. Dr. med. Santiago Ewig

Thoraxzentrum Ruhrgebiet

Standort Bochum,

Augusta-Kranken-Anstalt,

Klinik für Pneumologie,

Beatmungsmedizin und

Infektiologie

Bergstraße 26

44791 Bochum

ewig@augusta-bochum.de

\section{Einleitung}

Die Colitis ulcerosa ist eine primär chronisch entzündliche Darmerkrankung, die jedoch mit extraintestinalen Manifestationen einhergehen kann, wie z. B. Pyoderma gangraenosum, Erythema nodosum, Arthritiden, Augenkomplikationen wie Konjunktivitis, Iritis und Episkleritis, Leberdysfunktion, Pericholangitis, Fettleber, Venenthrombose sowie thromboembolischen Komplikationen. Eine pulmonale Mitbeteiligung ist erst seit der Veröffentlichung von Kraft u. Mitarb. in 1976 akzeptiert [1,7]. Die pulmonale Beteiligung bei chronisch entzündlichen Darmerkrankungen ist jedoch verglichen mit anderen Organbeteiligungen selten und wird möglicherweise auch deshalb häufig erst verzögert erkannt $[13,15]$. Stenosierungen der großen Atemwege sind unter den pulmonalen Beteiligungen eine Seltenheit, über die meist nur in Fallberichten geschrieben wird $[8,11,15]$.

Wir präsentieren den Fall einer 30-jährigen Patientin mit seit 10 Monaten persistierendem Husten infolge eines Befalles der großen Atemwege durch eine ulzeröse Colitis mit komplikativer Trachealstenose und Stenosierung beider Hauptbronchien.

\section{Fallbericht}

Bei der 30-jährigen Patientin wurde die Diagnose einer Colitis ulcerosa erstmalig im Januar 2002 gestellt, damals mit Befall des Kolons bis $25 \mathrm{~cm}$. Trotz einer topischen Therapie kam es zu einem schweren akuten Schub mit Pankolitis und Backwash-Ileitis einen Monat später. Daraufhin erfolgte die Einleitung einer systemischen Steroidtherapie. Eine ambulante Kontrollkoloskopie vom Juli 2004 zeigte einen makroskopischen Befall der aboralen Kolonhälfte, beginnend ab der linken Flexur. Es erfolgte eine Therapie mit Mesa- lazin $1 \times 1000 \mathrm{mg} / \mathrm{d}$, zusätzlich wurde die Patientin homöopathisch behandelt.

Aktuell klagte die Patientin seit Februar 2005 über einen anfangs trockenen, später produktiven Husten, der während einer Schwangerschaft erstmals aufgetreten war. Im Juli 2005 erfolgte die Entbindung von einer gesunden Tochter, von diesem Zeitpunkt an kam es zu einer Zunahme der Hustenattacken. In den letzten Wochen vor Aufnahme kam es zusätzlich zu Diarrhoen mit 10 bis 20 Entleerungen pro Tag mit gelegentlich peranalen Blutabgängen, verbunden mit rezidivierendem Fieber bis 39 C, Nachtschweiß, einer nicht quantifizierten Gewichtsabnahme sowie Gelenkschmerzen an Armen und Beinen.

Am Tag vor der Aufnahme im November 2005 begab sich die Patientin zum Lungenfacharzt. Dieser dokumentierte einen unauffälligen Auskultationsbefund sowie eine unauffällige Spirometrie und Ganzkörperplethysmographie: (IVC 2,40 l, $\mathrm{FEV}_{1}$ 2,201 (90\% des Solls), Resistance 0,18 kPa*sec pro Liter, ITGV 3,3l (128\% des Solls), eine unauffällige unspezifische bronchiale Provokation mit Carbachol, sowie eine mit 97\% Sättigung unauffällige Pulsoxymetrie. Es wurde eine ambulante Therapie mit Alvesco $160 \mu \mathrm{g}$ Dosieraerosol einmal täglich empfohlen. Am Tag darauf erfolgte die Einweisung durch die Hausärztin.

\section{Klinische Befunde}

Am 11. November 2005 zeigte sich die Patientin in einem befriedigenden Allgemeinzustand, untergewichtig ( $44 \mathrm{~kg}$ bei $159 \mathrm{~cm}$ Körpergröße), es bestand Fieber von 38,8 C, ein vesikuläres Atemgeräusch, das Abdomen war bis auf lebhafte Peristaltik unauffällig. Laborchemisch auffällig waren ein Hämoglobin von $8,0 \mathrm{~g} / \mathrm{dl}$, eine $\mathrm{LDH}$ von $222 \mathrm{U} / \mathrm{l}$, ein CRP von 14,0 mg/dl, darüber hinaus bestand eine Eisenmangelkonstellation (Fe $15 \mu \mathrm{g} / \mathrm{dl}$, Transferrin $183 \mathrm{mg} / \mathrm{dl}$, Transferrinsätti- 
gung 6\%, Ferritin mit $101 \mu \mathrm{g} / \mathrm{l}$ bei hohem CRP normal). In der Blutkultur wurde Streptococcus anginosus nachgewiesen.

Blutgasanalytisch fand sich bei Aufnahme eine Hyperventilation mit hochnormaler Oxygenierung $\mathrm{pO}_{2} 111,6 \mathrm{~mm} \mathrm{Hg}, \quad \mathrm{pCO}_{2}$ $31,2 \mathrm{~mm} \mathrm{Hg}, \mathrm{SO}_{2} 98,5 \%$, pH 7,49; lungenfunktionell - wie bereits ambulant - am 24.11. 2005 ein Normalbefund mit IVC 2,84 1 (85\% des Solls), FEV $_{1} 2,491$ (85\% des Solls), Tiffeneau-Index 87,7\%, TLC 4,3 1 (91\% des Solls), totaler Atemwegswiderstand 0,28 kPa*sec pro Liter (92\% des Solls), ITGV 2,95 l (114\% des Solls).

Die Röntgen-Thorax-Aufnahme vom 11.11.2005 zeigte einen Normalbefund.

Die CT-Thorax mit Kontrastmittel i.v. vom 17.11.2005 (O Abb. 1a u. b) zeigte eine Weichteilverdichtung der Trachea subglottisch dorsal sowie eine vermehrte weichteildichte Gewebsformation paratracheal und entlang beider Hauptbronchien.

Die Bronchoskopie vom 15.11. 2005 zeigte subglottisch von dorsal ein flaches Tumorbeet mit Lumeneinengung um 10 bis $25 \%$ ( Abb. 2). Der gesamte Tracheobronchialbaum war von zahlreichen, weißlichen, kleinen Noduli übersät mit sehr kontaktvulnerabler Schleimhaut ( Abb.3). Der linke Hauptbronchus war durch die zirkumferenten Noduli und ein erhebliches Schleimhautödem zu ca. 50\% eingeengt, ein ähnlicher Befund im rechten Hauptbronchus mit hier allenfalls 25-prozentiger Lumeneinengung (Abb.4). Die peripheren Segment-Ostien konnten aufgrund der Blutungsneigung und des trotz Sedierung starken Hustenreizes nicht eingesehen werden.

Histologisch handelte es sich in den Proben aus der Trachea um eine floride, chronische, granulierende, fokal auch ulzeröse Ent- zündung mit leichtgradiger Eosinophilie und fokaler Plattenepithelmetaplasie ohne Epitheldysplasie ( Abb.5). Das Bild entsprach einer Beteiligung des Respirationstraktes bei Colitis ulcerosa.

Eine Koloskopie vom 21.11. 2005 zeigte eine Pankolitis mit tieferen Ulcera im Rektum und Sigma, histologisch (Abb. 6) eine schwere ulzeröse Colitis mit Zerstörung der Kryptenarchitektur und Schleimdepletion.

\section{Verlauf}

Bei persistierenden Fieberschüben bis etwa 39 C sowie Nachweis von Streptococcus anginosus in der Blutkultur erfolgte eine antimikrobielle Behandlung mit Avalox $400 \mathrm{mg}$ täglich oral, zeitgleich wurde die antiinflammatorische Therapie mit Prednisolon $50 \mathrm{mg}$ täglich oral begonnen. Hierunter kam es umgehend zu einer Entfieberung und im weiteren Verlauf zu einer deutlichen Befundbesserung, Husten und Dyspnoe verschwanden; die Diarrhoen nahmen deutlich ab.

Eine immunsuppressive Therapie mit Azathioprin in steigender Dosierung bis zuletzt $100 \mathrm{mg}$ täglich wurde eingeleitet. Die Kontroll-Bronchoskopie ( Abb.7) nach 9 Tagen zeigte eine fast vollständige Rückbildung des entzündlichen Pseudotumors subglottisch dorsal sowie eine weitgehende Rückbildung der kleinnodulären Schleimhautveränderungen im gesamten Tracheobronchialbaum, es bestanden keine signifikanten Stenosierungen der Hauptbronchien mehr.
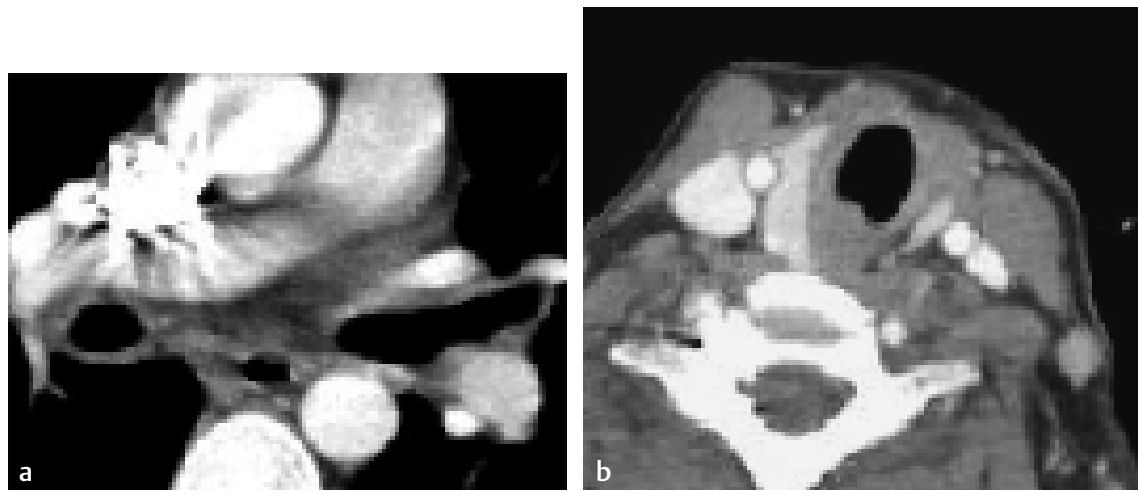

Abb. 1 a Gewebsformation entlang beider Hauptbronchien. b Weichteilverdichtung subglottisch

dorsal

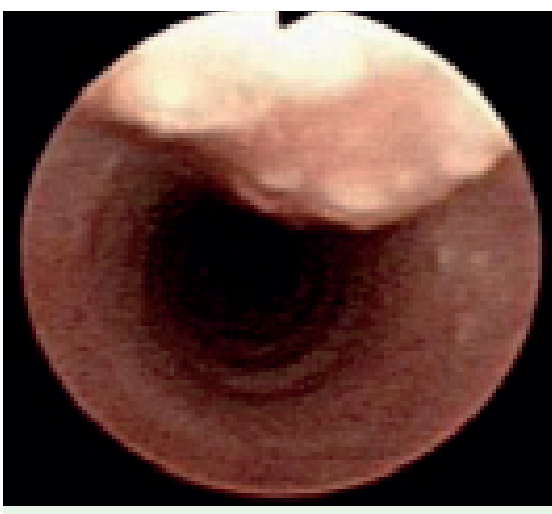

Abb. 2 Trachea subglottisch.

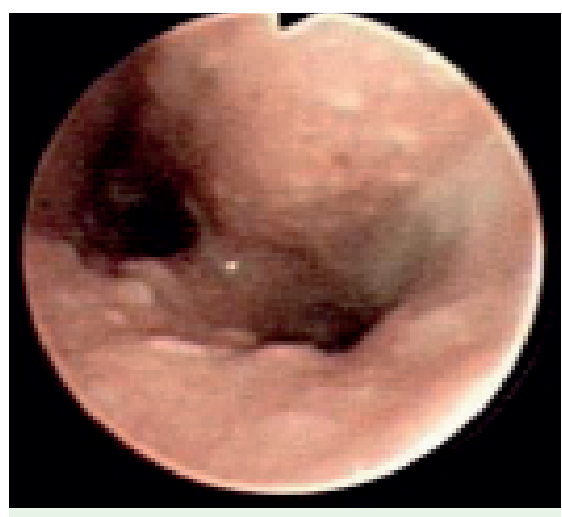

Abb. 3 Tracheobronchiale Noduli.

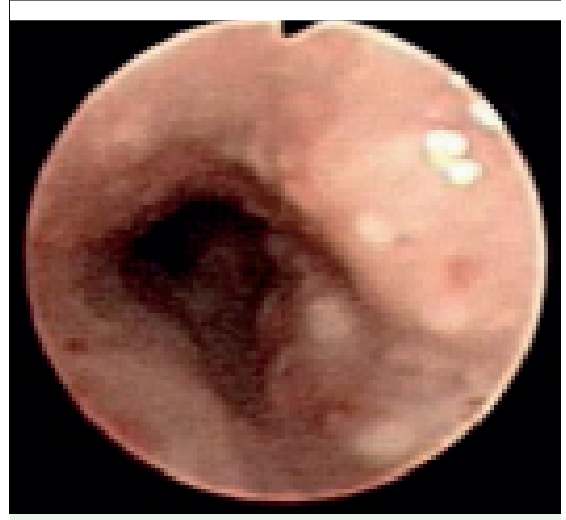

Abb. 4 Stenosierter linker Hauptbronchus. 


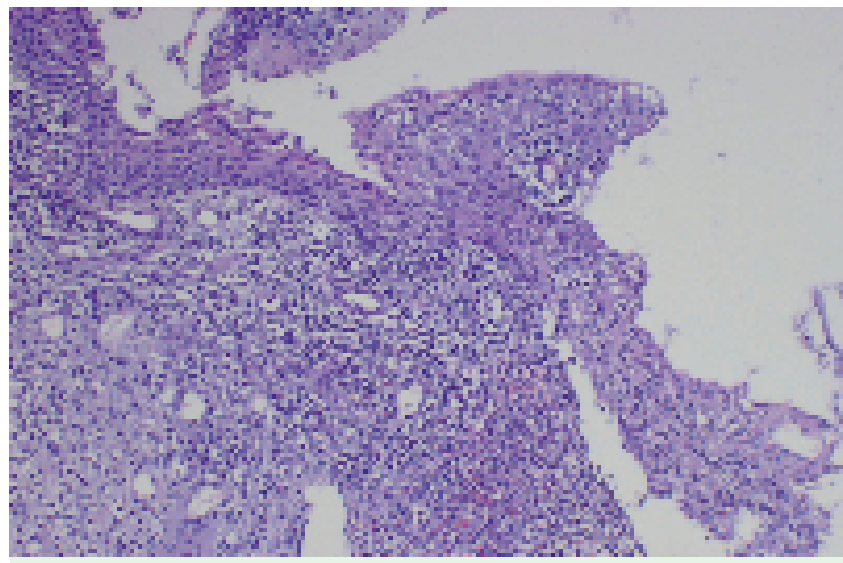

Abb. 5 Bronchiale Schleimhautbiopsie H\&E. 100fache Vergrößerung. Das bronchiale Epithel zeigt eine Plattenepithelmetaplasie und Infiltration durch polymorphonukleäre Leukozyten. Die Submucosa weist Granulationen, Lymphocyten, Neutrophile und eosinophile Leucozyten auf.

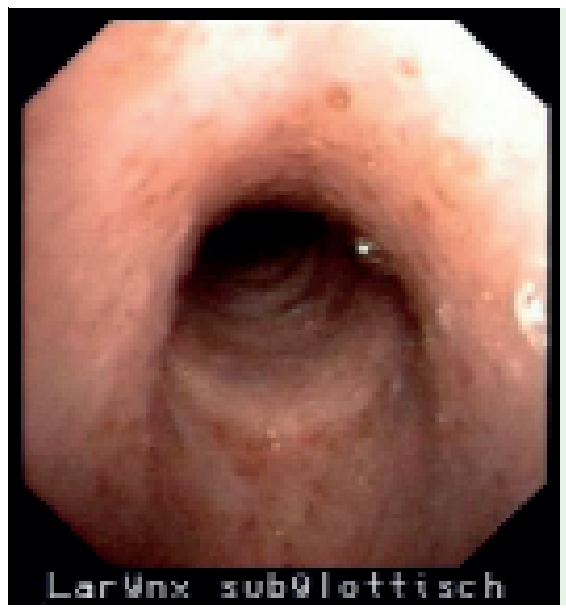

Abb. 7 Trachea subglottisch nach 9 Tagen Therapie.

\section{Diskussion}

Unsere Patientin stellte sich mit seit 10 Monaten bestehendem Husten mit unauffälligem Röntgen-Thorax-Bild sowie unauffälliger Lungenfunktionstestung vor. Die bronchoskopisch imponierende ausgeprägte Tracheobronchitis mit einem inflammatorischen Tumor subglottisch und Lumeneinengung um 10 bis $25 \%$ sowie eine Lumeneinengung beider Hauptbronchien um ca. $50 \%$ links und ca. $25 \%$ rechts durch ein ausgeprägtes Schleimhautödem sowie noduläre Schleimhautveränderungen entsprach histologisch einer floriden chronisch granulierenden, fokal auch ulcerösen Entzündung durch eine Beteiligung des Respirationstraktes bei Colitis ulcerosa.

Seit der Erstbeschreibung durch Kraft u. Mitarb. 1976 [7] sind eine Reihe von Publikationen über eine bronchopulmonale Beteiligung bei chronisch entzündlichen Darmerkrankungen erschienen. Diese scheinen bei Colitis ulcerosa häufiger als bei Morbus Crohn aufzutreten [1 - 3]. Berichtet wird über Stenosen der oberen Atemwege, Tracheobronchitis, Bronchiektasen, Bronchitis, Panbronchiolitis, nekrobiotische Herde, interstitielle Lungenerkrankungen und Pleuraergüsse $[1,6,7,11,12,15]$. Die größte Serie veröffentlichte Camus u. Mitarb. mit 33 Fällen, Higenbottam u. Mitarb. berichteten über 10 Fälle, Spira u. Mitarb. über 7 Fälle und Kraft u. Mitarb. über 6 Fälle.

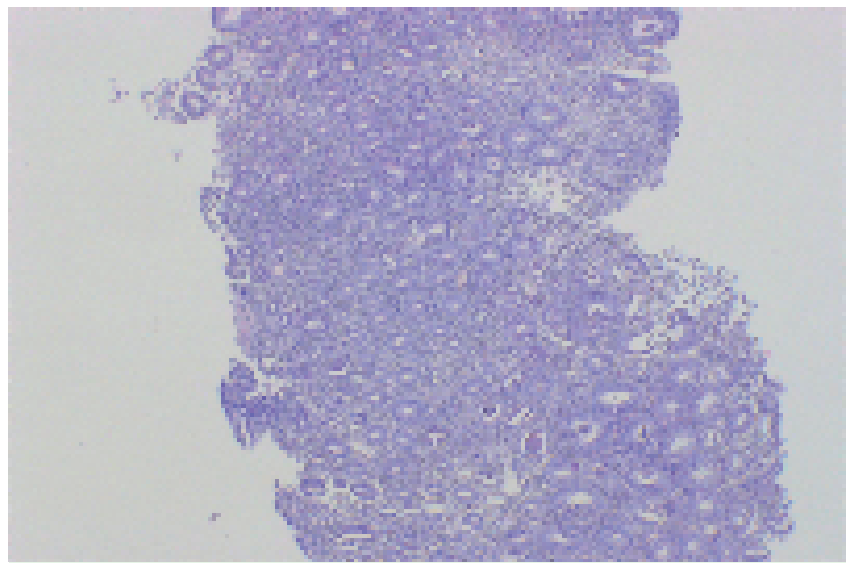

Abb. 6 Ulzerative Kolitis (Rektum-Biopsie H\&E. 40fache Vergrößerung): Irreguläre Krypten mit Krypten-Abszessen. Das zytoplasmatische Mucin der epithelialen Zellen ist vermindert.

Die Beteiligung der Atemwege im Rahmen der bronchopulmonalen Manifestationen einer chronisch entzündlichen Darmerkrankung ist relativ häufig [2]. Stenosierungen der großen Atemwege stellen dennoch eine Seltenheit dar. Die gleichzeitige ausgeprägte Beteiligung und Stenosierung von Trachea, beiden Hauptbronchien und Segmentbronchienabgängen mit entsprechenden endoskopischen und radiologischen Befunden, wie in unserem Fall, ist in dieser Form höchst selten und nur in Einzelfällen berichtet [14]. In einer Literaturübersicht über insgesamt 400 Fälle pulmonaler Manifestation von chronisch-entzündlichen Atemwegserkrankungen werden als häufigste Manifestationsform Bronchiektasen angegeben, als zweithäufigste Form eine chronische Bronchitis, jedoch nur bei 15 von 400 betroffenen Patienten eine Epiglottitis und Tracheobronchitis. Schwere und akut lebensbedrohliche Atemwegsstenosen sind eine Seltenheit $[4,11,15]$ (Übersicht bei [10]). Bei Obstruktionen der großen Atemwege finden sich gehäuft subglottische Stenosen durch inflammatorische Tumoren sowie Stenosen der großen Hauptbronchien, seltener auch Stenosen der Segmentbronchien (Übersicht bei [2]).

Radiologisch werden diese Veränderungen selten erfasst, so beschreiben Mahadeva u. Mitarb. [9] bei 17 Patienten mit chronisch-entzündlicher Darmerkrankung und bronchopulmonaler Beteiligung in 13 Patienten Bronchiektasen, in 5 ein „tree in bud“-Zeichen als Hinweis auf eine Bronchiolitis sowie in 2 Patienten fibrosierende Veränderungen. Bronchialwandverdickungen wurden nicht beobachtet. Garg u. Mitarb. [5] berichten in einer retrospektiven Untersuchung mit HR-CT bei 3 von 7 Patienten mit Colitis ulcerosa und Symptomen von Husten und rezidivierenden respiratorischen Infektionen über Bronchialwandverdickungen und Atemwegsstenosen.

Typisch für die seltene Manifestationsform in unserem Fall litt unsere Patientin monatelang unter Symptomen der Atemwege, ohne dass die zugrunde liegende Erkrankung erkannt wurde. Diese Diagnoseverzögerung erklärt sich auch durch ein normales Röntgen-Thoraxbild sowie eine normale Lungenfunktionstestung, selbst zu einem Zeitpunkt zunehmender Krankheitssymptome und bronchoskopisch ausgeprägter Befunde.

Bei unserer Patientin konnten wir die Beteiligung der Atemwege und gleichzeitige Exazerbation der Colitis ulcerasa im Dickdarm histologisch eindrucksvoll nachweisen. Das bekannt gute Ansprechen auch der pulmonalen Manifestation der Colitis ulcero- 
sa auf Prednisolon $[1,6,15]$ zeigte sich auch bei unserer Patientin. Bei subjektiv deutlicher Beschwerdelinderung fand sich in der Kontroll-Bronchoskopie nach 9 Tagen Therapie nur noch ein geringer Restbefund, die Stenosierungen von Trachea und beiden Hauptbronchien waren praktisch nicht mehr vorhanden.

Die Bakteriämie durch Streptococcus anginosus geht am ehesten von oral aus. In unserem Fall konnte eine Quelle der Bakteriämie nicht identifiziert werden, eine bronchiale Quelle ist unwahrscheinlich.

Unser Fall demonstriert eindrücklich, dass bei Patienten mit chronisch entzündlichen Darmerkrankungen und gleichzeitiger respiratorischer Symptomatik an eine Lungenbeteiligung gedacht werden muss und entsprechend eine umfassende bildgebende und bronchologische Diagnostik indiziert ist. Das gute therapeutische Ergebnis unterstreicht die Bedeutung einer frühen und zielführenden Diagnostik.

\section{Literatur}

1 Camus P, Piard F, Ashcroft T et al. The lung in inflammatory bowel disease. Medicine 1993; 72: 151-183

2 Camus P, Colby TV. Respiratory manifestations in ulcerative colitis. Eur Resp Mon 2006; 34: 168 - 183

3 Chenivesse C, Bautin N, Wallaert B. Pulmonary manifestations in Crohn's disease. Eur Resp Mon 2006; 34: 151 - 167

4 Cross DL, Scudder DD. Airway obstruction in ulcerative colitis. South Med J 1997; 90: 249-250
5 Garg K, Lynch DA, Newell JD. Inflammatory airways disease in ulcerative colitis: CT and high-resolution CT features. J Thorax Imaging 1993; 8: 159- 163

6 Higenbottam T, Cochrane GM, Clark TJ et al. Bronchial disease in ulcerative colitis. Thorax 1980; 35: $581-585$

7 Kraft SC, Earle RH, Roesler $M$ et al. Unexplained bronchopulmonary disease in patients with inflammatory bowel disease. Arch Intern Med 1976; 136: $454-459$

8 Lamblin C, Copin M-C, Billaut C et al. Acute respiratory failure due to tracheobronchial involvement in Crohn's disease. Eur Respir J 1996; 9: $2176-2178$

9 Mahaveda R, Walsh G, Flower CDR et al. Clinical and radiological characteristics of lung disease in inflammatory bowel disease. Eur Resp J 2000; $15: 41-48$

10 Marvisi M, Bassi E, Civardi G. Pulmonary involvement in inflammatory bowel disease. Current Drug Targets - Inflammation \&Allergy 2004; 3: $437-439$

11 Rickli H, Fretz C, Hoffman $M$ et al. Severe inflammatory upper airway stenosis in ulcerative colitis. Eur Respir J 1994; 7: 1899-1902

12 Spira A, Grossman R, Balter M. Large airway disease associated with inflammatory bowel disease. Chest 1998; 113: 1723 - 1726

13 Storch I, Sachar D, Katz S. Pulmonary manifestations of inflammatory bowel disease. Inflammatory Bowel Diseases 2003; 9: 104-115

14 Ward H, Fisher KL, Waghray R et al. Constrictive bronchiolitis and ulerarative colitis. Can Resp J 1999; 6: 197 -200

15 Wilcox $P$, Miller R, Miller G et al. Airway involvement in ulcerative colitis. Chest 1987; 92: $18-22$ 